\title{
Prescribing for the elderly
}

\author{
M R BLISS
}

Generally, prescribing in Britain today is in a mess: the annual expenditure of the NHS on drugs is $£ 500 \mathrm{~m}$; expenditure on drugs not taken, "non-compliance," is $£ 20 \mathrm{~m} .{ }^{1}$ Prescription rates are as follows:

$55 \%$ of the whole male population receive at least one prescription a year ${ }^{2}$;

$65 \%$ of the whole female population receive at least one prescription a year ${ }^{2}$;

$33 \%$ of all women aged $45-59$ receive psychotrophic drugs ${ }^{2}$;

$37 \%$ of all women aged 75 and over receive psychotrophic drugs $^{2}$;

$75 \%$ of the whole population aged 75 and over receive drugs of some kind ${ }^{2}$; two-thirds receive one to three drugs, and onethird four to six drugs simultaneously ${ }^{3}$;

$25 \%$ of all adults are taking medicines first prescribed more than one year previously ${ }^{4}$;

$75 \%$ of prescriptions are repeat prescriptions ${ }^{4}$;

$50 \%$ of patient's bottles seen in a geriatric outpatient clinic have no dosage directions (personal observation);

$80 \%$ of patients admitted to geriatric wards are receiving drugs $^{3}$; for most of these patients, and for patients seen in geriatric outpatient clinics, it is impossible to be certain what the drugs are; and

$10 \%$ of admissions to geriatric wards are due to iatrogenic disease caused by drugs. ${ }^{3}$

The need for a prescription for every ill felt by both doctors and patients is not new. Readers of George Eliot's Middlemarch will recall the struggles of young Dr Lydgate and the unpopularity that he brought on himself, with both his colleagues and his patients, by trying to resist the demand for useless bottles of physic. In this respect doctors are more culpable than patients. Studies $^{5}$ have shown that, whereas $80-90 \%$ of doctors expect

Department of Geriatric Medicine, Hackney Hospital, London E9 6BE

M R BLISS, MB, MRCP, consultant geriatrician patients to want prescriptions, only $30-50 \%$ of patients actually do; the remainder would prefer an examination, advice, and reassurance, but are frequently given a prescription instead.

In the past placebos, or the bona-fide medicines of the day, were often unpleasant, but they seldom had the powerful physiological actions of today's drugs, nor were they so freely available. Today the elderly are the main victims of modern drugs and the system by which they are administered. The reasons for this are: (1) multiple pathology of the elderly, (2) polypharmacy, (3) increased sensitivity of the elderly to drugs and side effects, (4) doctors' lack of training in geriatric prescribing, (5) unsuitable drug packaging and instructions, (6) poor supervision of elderly patients, and (7) dual prescribing systems in hospitals and in general practice, which prevent doctors from being fully responsible for their own prescribing.

\section{Multiple pathology and polypharmacy}

The multiple diseases of the elderly cause them to consult their doctors frequently. Doctors, encouraged by the pharmaceutical companies, are tempted to try to treat each symptom, often without giving sufficient thought to its causes. The resulting polypharmacy itself can prevent drugs from acting properly or exacerbate their side effects.

\section{Increased sensitivity of the elderly to drugs and side effects}

The increased sensitivity of the elderly to drugs and side effects is due to a variety of causes, including reduced body weight and plasma volume. Elderly patients, especially sick patients, often also have a reduced plasma albumin concentration. Certain drugs-for instance, warfarin, phenytoin, sulphonureas, salicylates, and phenylbutazone-are bound to albumin in their transport round the body. In the bound state they are pharmacologically inactive, so that only the small amounts of 
free drugs are responsible for their effects. If two or more of the binding drugs are given together, however, they will displace each other from the available albumin, especially if this is reduced, causing variable pharmacological reactions from the same doses. ${ }^{8}$

Other drugs, such as barbiturates and diazepam, are absorbed by body fats, ${ }^{\circ}$ causing prolonged action in obese, elderly patients.

The two drug elimination pathways, via the liver and via the kidney, are both impaired in the elderly. There is reduced extraction of some drugs, such as propranolol, from the blood stream by the liver, and reduced enzyme induction, affecting the breakdown of, for example, barbiturates and warfarin. ${ }^{\circ}$ In the kidney reduced renal clearance of drugs, such as digoxin, penicillin, aminoglycosides-for instance, streptomycin, gentamycin-and chlorpropamide, parallels that of creatinine. Creatinine clearance in even healthy elderly people is reduced by about $30 \%$ of that in youth ${ }^{7}$ and still more in renal failure. Elimination of these drugs is therefore always impaired in the elderly, and, unless this is allowed for in dosage, may cause serious toxicity.

Rarely an increased reaction to a drug in the elderly seems to be due to an increased sensitivity of the target organ itself-for example, the increased response of the aging brain to "normal" blood concentrations of diazepam.

\section{Doctors' lack of training in geriatric prescribing}

The British National Formulary has a special section on paediatric prescribing but no similar section for geriatric patients. This would be less easy to define, as the elderly differ more in their need for, and ability to handle, drugs than children, but nevertheless, such a section would probably be a great help to practitioners prescribing for patients over 75 .

Almost all patients of this age require some modification of treatment-for example, some drugs, especially potentially toxic drugs, such as digoxin, warfarin, levodopa, phenothiazines, and night sedatives, need to be given in reduced doses often amounting to half of those recommended for younger patients; other drugs, such as diuretics, need to be used with deliberation; the potential effect of different types of diuretics on potassium retention and hypotension, their speed of action, and the ability of patients to get to the lavatory, and therefore the possible effect of the diuresis on incontinence, must all be considered.

Still other drugs such as hypertensives, should be seldom, if at all, used for the elderly. These are scarcely ever necessary, whatever the blood pressure, and side effects such as hypotension and depression are severe.

Similarly, drugs used specifically to treat dizziness-for instance, prochlorperazine (Stemetil)_are far more likely to do harm than good. Prochlorperazine should never be used except for true vertigo, characterised by a sensation of rotation, which is extremely rare in the elderly. Probably more patients are admitted to hospital suffering from the side effects of this drug-hypotension and Parkinsonism-than from those of any other.

Cerebral and peripheral vasodilators and stimulators of anaerobic metabolism-for example, dihydroergotoxine (Hydergine), cyclandelate (Cyclospasmol), and Naftidrofuryl (Praxiline) -are another group of drugs that are virtually ineffective in the late $70 \mathrm{~s}$ and $80 \mathrm{~s}$, and often cause serious reactions such as nausea, bradycardia, flushing, diarrhoea, headache, etc.

Antidepressants, such as amitriptyline, doxepin, mianserin, and antirheumatic drugs usually do need to be given in doses similar to those used for young patients to be effective, but the doctor has to be particularly alert for side effects-half of elderly patients taking indomethacin develop gastric bleeding. It is essential to be sure that these drugs are really necessary, and then to review the patient frequently to assess their effect and watch for adverse reactions. If a patient has not responded to an antidepressant within three weeks it should be stopped. Antidiabetic drugs have also to be given in adequate doses when required, but many mild diabetics in this age group often need drugs only at times of stress, such as intercurrent illness. Acceptably low blood sugar concentrations, up to $10 \mathrm{mmol} / \mathrm{l}$, are often maintained without even the help of a diet (itself an unpleasant and often unsuccessful imposition on an old person). If a sulphonurea is necessary, tolbutamide, or preferably glibenclamide, which may be given once a day, should be used instead of chlorpropamide. Chlorpropamide has a long half life that is dangerously increased by reduced renal clearance in the elderly.

Diazepam and barbiturates should not be prescribed for the elderly at all. Diazepam has a half life in hours as lnng as the patients' age in years, and both have a toxic effect on the aging brain, causing increased confusion and drowsiness.

Side effects and interaction of drugs must be uppermost in the minds of all doctors prescribing for the elderly, and are best avoided by reducing the number of prescriptions to the absolute minimum, using no drugs at all if possible, and reviewing the patient frequently. All drugs should be stopped if they are not helping the patient, and most drugs, even those that are apparently" being effective, should be tailed off and stopped from time to time to see if they are still necessary.

Essential drug regimens should be made as simple as possible -for example, one heart tablet, one water tablet, and one diabetic tablet to be taken together before breakfast. If antirheumatic drugs are necessary, those that can be given twice a day-for instance, benorylate, sulindac, or naproxen-are easier for patients to take and more likely to tide patients over the night than drugs such as ibuprofen or aspirin, which have to be taken every four to six hours for the same effect. ${ }^{8}$

\section{Drug packaging and instructions}

The drug must be provided in a form that the patient can swallow, and in a suitable package with precise, readable instructions.

Patients are used to dealing with screw-topped bottles and are likely to manage these better than ones with press-on caps. Most elderly patients are unable to open child-proof containers, which therefore should not be used. The bottles should be of clear glass whenever possible so that the tablets and their colours are readily recognisable. Elderly people find blister packs difficult to understand and use."

Many pharmacists now type the labels on their bottles, but even plainer, fuller directions, such as those suggested by Das and Williams ${ }^{10}$ (fig 1), are needed. Instead of relying on instructions written on bottles, which necessarily have to be small, some practitioners supply elderly patients, or patients with complicated drug regimens, with separate instruction

\begin{tabular}{|c|c|c|c|}
\hline \multirow{2}{*}{$\begin{array}{l}\text { MAME } \\
\text { TIME } \\
\end{array}$} & Ms Juliet Montague & \multicolumn{2}{|c|}{ DATE $27-2-75$} \\
\hline & NAME OF DRUG & DESCRIPTION OF DRUG & DOSE \\
\hline \multirow[t]{3}{*}{$7 \mathrm{am}}$. & St Joseph's Minture & Blue medicine for pain & One enve \\
\hline & PREDNISOLONE & mick tablet for appetite & 1 tablet \\
\hline & NAVIDREX - K & Yellow tablet for swollen ankles & 1 tablet \\
\hline $11 \mathrm{am}$. & St Josepth's Mixture & & One some \\
\hline \multirow[t]{2}{*}{$3 \mathrm{pm}}$. & St. Joseptr's Mixture & & One eme \\
\hline & PREDNISOLONE & & I tablet \\
\hline $7 \mathrm{pm}$. & st. Joseph's Mixture & & Ongeosine \\
\hline \multirow[t]{3}{*}{$11 \mathrm{pm}$} & St Josephis Mixture & & one evive \\
\hline & DORBANEX & Yelnow hauid for constipation & Ong Bme \\
\hline & PREONISONONE & & 1 tablet \\
\hline
\end{tabular}

FIG 1-Drug package labelling, with detailed dose instructions. ${ }^{10}$ 
sheets such as those used by St Joseph's Hospice, London ${ }^{11}$ (fig 2). Cards such as these should be available in all outpatient departments, and on wards, for the use of patients leaving hospital.

Several days before discharge from the geriatric assessment unit of the Southern General Hospital, Glasgow, ${ }^{12}$ patients are supplied with the bottles of tablets and medicines that they are going to take at home so that they can learn to give themselves their own medication under supervision.

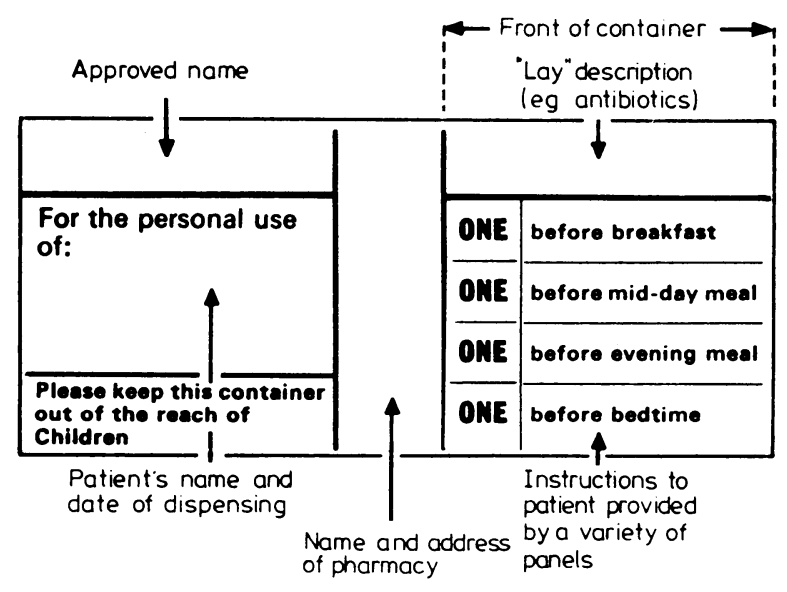

FIG 2-Drug instruction card. ${ }^{11}$

Time and again it has been shown that the ability of elderly patients to take their tablets reliably depends on the amount of explanation and encouragement received from their doctors, on the number of drugs that they are expected to take at one time, ${ }^{13}$ on the frequency of the doses ${ }^{14}$ and preparation of the drugs, and on the instructions supplied. It is essential to remember that some patients will not be able to read the instructions, and others will not be able to understand or carry them out reliably. Confused patients must have their drugs administered by a third person-for instance, a spouse, home-help, or district nurse - or not be given medication.

However good their labelling may be, pharmacists' directions on their bottles have to depend on doctors' prescriptions. Half the prescriptions provided in general practice nowadays have "as directed" or "as before" as the only dose direction. The resulting labels on the bottles make it impossible for patients or their helpers to know if they are taking their tablets correctly. These prescriptions, which are causing as much concern to pharmacists as they are to patients, are almost always written by receptionists. Receptionists, who have no medical training and usually no training at all, write prescriptions at the patients' request, either by copying them from patients' record cards, from a hospital discharge letter, from the patients' bottles, from a patients' copy in a letter of the wording on his bottles, or from dictation by the patient on the telephone. As doses are often absent and, in any case, receptionists do not know the meaning of the symbols bd, tds, prn, etc, they either omit doses (and sometimes also strength so that a prescription may simply read, for example, "Aldomet 100") or, more commonly, they write "as directed" or "as before." Doctors sign the foot of these prescriptions in sheaves at the end of their surgeries without time to check them. ${ }^{15}$ Pharmacists are legally obliged to transfer whatever is written on the prescription on to their labels, and so the system perpetuates itself.

\section{Poor supervision}

The sicker elderly patients are, the more likely they are to be receiving large numbers of drugs and the less likely they are to be able to visit the surgery. They have to rely either on visits from the doctor, or on the post, or on the help of another person to obtain their drugs. General practitioners vary in their willingness to visit elderly patients. By far the majority of housebound patients obtain their prescriptions through repeat prescribing systems. They may not be seen by their doctors for months, or possibly years, on end, sometimes with severe consequences. For example, one of my patients, a chairbound woman who had been receiving butazoladine for arthritis for several years without being seen by her doctor, was found to have a haemoglobin concentration of $6 \mathrm{~g} / \mathrm{dl}$. Another patient who had been treated with metaclopamide (Maxolon) for five years after a single attack of cholecystitis developed an irreversible dystonia.

Patients are often unsure about what drugs they need. Sometimes they are afraid that their doctor will be angry if they fail to keep on sending for the drug that he has prescribed for them. They do not like to complain about side effects, and think that they should not trouble the doctor by asking him to see them to give them prescriptions personally. They are also anxious about running out of tablets because the surgery may be closed, or they may have nobody to send for their drugs; so they tend to order more drugs on repeat prescription than they need. They are often uncertain what to do with the tablets when they arrive, especially if the bottles are inadequately labelled. They may feel better and think they no longer need them, or they may find that the drug or its side effects are too powerful for them to be able to continue taking it. Sometimes the treatment itself is changed, either by their own doctor, or another doctor whom they may be seeing elsewhere. As a result their bottles tend to accumulate. As any practitioner or consultant visiting patients at home knows, patients receiving repeat prescriptions invariably have dozens of half-filled bottles lining their bedside tables, mantelshelves, and cupboards.

\section{Dual prescribing systems in hospital and in general practice}

Much money and effort have been put into studying the reasons for patient non-compliance, but doctors and research workers seem blind to the deficiencies in the present system of prescribing, which makes it impossible for doctors to prescribe drugs reliably or for patients to obtain them and take them properly.

At present pharmacists are reimbursed for drugs prescribed by general practitioners, and for drugs prescribed in hospitals, out of two separate NHS funds. Practitioners' prescriptions on FP 10 forms are paid for out of an unlimited general prescribing fund. Hospital prescriptions, whether they are written on hospital prescription charts or on FP 10 (HP) forms, and whether they are dispensed in the hospital pharmacy or outside, are paid for out of the general budget for that particular hospital. Recent curbs on hospital spending have accentuated this anomaly by causing management committees to try to save money by limiting the amount of medicine prescribed by hospital doctors for outpatients, or for patients leaving hospital. Doctors are directed to ask patients' practitioners to prescribe the recommended drugs for them instead.

Consider a patient being discharged from hospital. $\mathrm{He}$ is given a limited supply of drugs, usually enough for one week, to take home with him. At the same time a cyclostyled letter is sent to his general practitioner telling him what drugs the patient is taking. This list is often copied by a nurse from the patient's ward treatment chart, and may contain unnecessary drugs such as vitamins and sleeping pills that are not checked by the house officer when he signs it. The practitioner is asked to provide the patient with further supplies. He, in his turn, is likely to hand this letter to his receptionist for repeat prescribing. Meanwhile the patient has been told to go and see his doctor to get his drugs. A study by Deacon et $a l^{16}$ showed that, of patients being prescribed drugs on discharge from a district general hospital, $20 \%$ had not contacted their general prac- 
titioner at the end of one month. There was no correlation between this failure and the type of drugs the patient had been prescribed, which included cardiovascular, antibiotic, and psychotrophic drugs, and hormones, but most patients who did contact the surgery were those who had had similar drugs prescribed for them before their admission to hospital-that is, they were the long-standing patients who swing back into the routine of obtaining repeat prescriptions. Often, because they have not received instructions to the contrary, patients send for the drugs that they were receiving before admission to hospital, as well as the new drug prescribed for them on discharge. ${ }^{17}$

After discharge, patients are often given a follow-up appointment as an outpatient. Here, as at all outpatient appointments, it is difficult to be sure what treatment the patient is receiving. Patients may not like to admit that they have run out of tablets. Many elderly patients do not know what their medicines are. If they are told to bring their bottles these often represent only a selection of those that they think important, and doses on them are often missing. It is impracticable to telephone the practitioners to ask about the treatment of almost every patient and in any case the reply is often vague. The clinic doctor has to assess the patient and decide about treatment with no real idea about what drugs he is already receiving. Then, again, owing to present instructions, he is unable to prescribe sufficient drugs to last the patient until his next outpatient appointment. He may either tell the patient to get all his supplies from his doctor, or, so as not to delay the start of his treatment, he may prescribe enough for one week for the patient to take until he can visit his doctor or obtain a repeat prescription. Patients liable to prescription charges may thus have to pay the present high prices of their medicines twice in one month or go without. Fortunately, elderly patients are exempt from charges, but they too are dismayed by being told that they must get their drugs from their practitioners. Many housebound patients protest that they never see their doctor and do not know whom to send to the surgery. Some also remark that they do not like the bottles of medicine that they get from the surgery because they have no instructions.

After the visit the clinic doctor usually writes to the patient's practitioner telling him his findings and what treatment he recommends. At best these letters are unlikely to reach the general practitioner within a week, and sometimes they may be delayed for as long as a month, or they may not be written at all. The quality of the letters vary. The description of the patient's drug treatment is often particularly sketchy even though this part of the letter is in effect a directive to the general practitioner. Drug information cards, which are provided by some outpatient departments to give to patients themselves to take to their practitioners, also tend to be poorly completed or ignored, mainly because copying out prescriptions, in effect, three times for each patient, is prohibitively time consuming in a busy clinic. In any case practitioners justly resent spending their time writing other doctors' prescriptions for them, sometimes for treatments with which they may not even agree. They are often brusque with patients when they present their requests and whenever possible relegate the whole unpleasant task to their repeat prescribing systems.

\section{Patient prescription booklets}

There can be no hope of reforming prescribing until the separate funding of general practice and hospital drugs is removed. Every effort should be made to achieve this as soon as possible. Once this is done it should be possible to devise a unified system of prescribing that ensures that doctors can be responsible for writing their own prescriptions, and at the same time be fully aware of what drugs the patient is already receiving from other sources.

One way of doing this would be to provide a patient prescription booklet for outpatients similar to the inpatient hospital prescription chart. ${ }^{18}$ This would be kept by the patient himself, who would take it to all medical appointments in hospital and in general practice to be used by all doctors, including hospital doctors writing prescriptions for him as an outpatient. The booklet would be presented to the pharmacist for dispensing and returned to the patient with his drugs. Each book could last patients for two years or more. It would contain a complete record of the drugs, including strengths, doses, and amounts supplied to that patient during that time. Provision could be made for a doctor's single signature to authorise up to two repeat prescriptions-that is, three months' supply of drugswhich could be dispensed without the patient having to revisit the surgery. Thereafter, a new prescription would have to be obtained, normally only after the patient had seen the doctor again. This would greatly reduce the total number of prescriptions each doctor would be required to write. It should therefore be possible to insist that all prescriptions be written wholly in the doctor's hand. Receptionist prescribing, which, however well controlled, is always liable to misuse, would be eliminated.

The existence of the drug booklet charts would make it easier for doctors and pharmacists, and helpers such as district nurses, to know what drugs and in what amounts were being dispensed for patients. It would enable patients themselves to have a better understanding of their own drugs. The booklets would also be a boon to staff in homes for old people, who at present have great difficulty in distributing the large numbers of drugs prescribed for residents solely by means of the inadequate labels on their bottles. ${ }^{19}$

\section{References}

${ }^{1}$ Anonymous. Non-compliance; does it matter? $\mathrm{Br} \mathrm{Med} \mathcal{F}$ 1979;ii:1168.

2 Skegg DCG, Doll R, Perry J. Use of medicines in general practice. Br Med f 1977 ; i:1561-3.

3 Williamson J. Prescribing problems in the elderly. Practitioner 1978;220: 749-55.

4 Dunnell K, Cartwright A. Medicine takers, prescribers and hoarders. London: Routledge and Kegan Paul, 1972.

5 Stimson GV. Doctor-patient interaction and some problems for prescribing. $\mathcal{F}$ Coll Gen Pract 1976;26,suppl $1: 88-96$.

- Cook P. How drug action is altered in the elderly. Geriatric Medicine 1979 April:45-6.

7 Castledon CM. Prescribing for the elderly. Prescriber's fournal 1978;18: 90-4.

${ }^{8}$ Hart FD, Taylor RT, Huskisson EC. Pain at night. Lancet 1970;i:881-4.

- Gibson IJM. Are drugs in the right containers for your elderly patients ? Modern Geriatrics 1978 March: 39.

10 Das BC, Williams A. Aids to drug compliance. Br Med f 1978;i:789-90.

11 Lamerton R. Home care of the dying. St Bartholomew's Hospital fournal $1975 ; 79$ :No 6.

12 Baxendale C, Gourlay M, Gibson IJM. A self medication retraining programme. $B r$ Med $71978 ; \mathrm{ii}: 1278-9$.

13 Parkin DM, Henney CR, Quirk J, Crooks J. Deviation from prescribed drug treatment after discharge from hospital. Br Med $\mathcal{F}$ 1976;ii:686-8.

14 Gatley MS. To be taken as directed. $\mathcal{F} R$ Coll Gen Pract 1968;16:39-44.

15 Bliss MR. Prescribing in general practice-views of a locum in Kent. Lancet 1978; ;i :248-9.

16 Deacon SP, Hammond L, Thompson B. Drug supply requirement for patients discharged from hospital. $\mathrm{Br}$ Med $\mathcal{f} 1978 ; \mathrm{ii}: 555$.

17 Drury VSM, Wade OL, Woolf E. Following advice in general practice. f $R$ Coll Gen Pract 1976;26:712-8.

18 Bliss MR. Rationalising out-patient prescribing. Practitioner 1978;220: 357-60.

10 Tallet ER. Management of medicines in residential homes. $\mathrm{Br} \mathrm{Med} \mathfrak{f}$ $1977 ; \mathrm{ii}: 641$.

(Accepted 19 May 1981) 